\title{
Scattering of backward spin waves in a one-dimensional magnonic crystal
}

\author{
A. V. Chumak, ${ }^{1, a)}$ A. A. Serga, ${ }^{1}$ B. Hillebrands, ${ }^{1}$ and M. P. Kostylev ${ }^{2}$ \\ ${ }^{1}$ Fachbereich Physik and Forschungszentrum OPTIMAS, Technische Universität Kaiserslautern, \\ 67663 Kaiserslautern, Germany \\ ${ }^{2}$ School of Physics, University of Western Australia, Crawley, Western Australia 6009, Australia
}

(Received 28 May 2008; accepted 1 July 2008; published online 17 July 2008)

\begin{abstract}
Scattering of backward volume magnetostatic spin waves from a one-dimensional magnonic crystal, realized by a grating of shallow grooves etched into the surface of an yttrium iron garnet film, was experimentally studied. Rejection frequency bands were clearly observed. The rejection efficiency and the frequency width of the rejection bands increase with increasing groove depth. A theoretical model based on the analogy of a spin-wave film waveguide with a microwave transmission line was used to interpret the obtained experimental results. (C) 2008 American Institute of Physics.
\end{abstract}

[DOI: 10.1063/1.2963027]

Artificial media with periodic lateral variation in their magnetic properties, so-called magnonic crystals, ${ }^{1}$ are the magnetic analog to photonic and sonic crystals and are suitable for operation in the microwave frequency range. Spectra of spin-wave excitations in such structures are considerably modified with respect to uniform media and exhibit features such as full band gaps, ${ }^{2}$ where spin waves are not allowed to propagate. Due to the wide tunability of their properties, these periodical structures offer excellent potential for the investigation of linear and nonlinear spin-wave dynamics. ${ }^{3}$

Both ferromagnetic and ferrite media can be artificially structured for the fabrication of magnonic crystals of different dimensionalities. We have chosen a single-crystal ferrite film of yttrium iron garnet (YIG) as the magnetic material because of the extremely small spatial damping of spin waves in monocrystalline YIG films. Experimentally this allows one to observe spin-wave propagation over a distance of the order of $1 \mathrm{~cm}$. Furthermore, we have focused on onedimensional structures because they allow for operation at only one eigenmode of the film. The latter is determined by the angle between the wave propagation direction and the external magnetic field orientation. Previously, only the surface magnetostatic waves ${ }^{4}$ (wave propagation direction is perpendicular to the magnetic field applied in the film plane) and forward magnetostatic waves ${ }^{5}$ (external field is oriented perpendicular to the film plane) were studied in such periodical structures. ${ }^{3,6-8}$ However, nowadays special attention is focused on spin waves of backward type, so-called backward volume magnetostatic waves (BVMSWs), due to their special benefits both for research on nonlinear spin-wave dynamics $^{9,10}$ and application. ${ }^{11,12}$ Here we present experimental and theoretical results on the scattering of BVMSW from a structure with periodic changes in the film thickness.

To fabricate the magnonic crystal, a 5.5- $\mu \mathrm{m}$-thick YIG film was used, which was epitaxially grown along the (111) crystallographic axis. Hot orthophosphoric acid etching and photolithography were used to prepare the grooves. The lithography was based on a standard photoresist AZ 5214E baked by UV irradiation, which is stable against hot $160{ }^{\circ} \mathrm{C}$ orthophosphoric acid. The mask had $N=20$ parallel lines $w$ $=30 \mu \mathrm{m}$ in width and spaced $270 \mu \mathrm{m}$ from each other, so

\footnotetext{
a) Also at Taras Shevchenko National University of Kiev, Ukraine. Electronic
} mail: chumak@physik.uni-kl.de. the lattice constant was $a=300 \mu \mathrm{m}$. The grooves were perpendicularly oriented with respect to the spin-wave propagation direction. In order to study the dependence of grating characteristics on the groove depth $\delta$, the grooves were etched in $100 \mathrm{~nm}$ steps from $100 \mathrm{~nm}$ to $1 \mu \mathrm{m}$. The groove depth was controlled by the etching time and measured using a profilometer. Anisotropic chemical etching caused by the YIG crystallographic structure was observed: the speed of etching parallel to the film plane was approximately ten times larger than that in the perpendicular direction, so the final groove depth profile along the direction of wave propagation had a trapezoidal shape.

Two microstrip antennas placed $8 \mathrm{~mm}$ apart, one in front of the grating and one behind it, were used to excite and receive the dipolar spin waves (see Fig. 1). A bias magnetic field of 1845 Oe was applied in the plane of the YIG film stripe along its length and parallel to the direction of spinwave propagation. Thus the conditions for BVMSW propagation are given. The microwave signal power was equal to $1 \mathrm{~mW}$ to avoid any nonlinear processes.

The experimental BVMSW transmission characteristics for the unstructured film as well as for the grating with $\delta$ $=300,600$, and $900 \mathrm{~nm}$ are shown in Fig. 2(a). Figure 2(b) demonstrates groove structure induced losses calculated as a difference between the transmission characteristics for the unstructured and structured films. The dotted straight line indicates the limit of the dynamic range of the experimental setup.

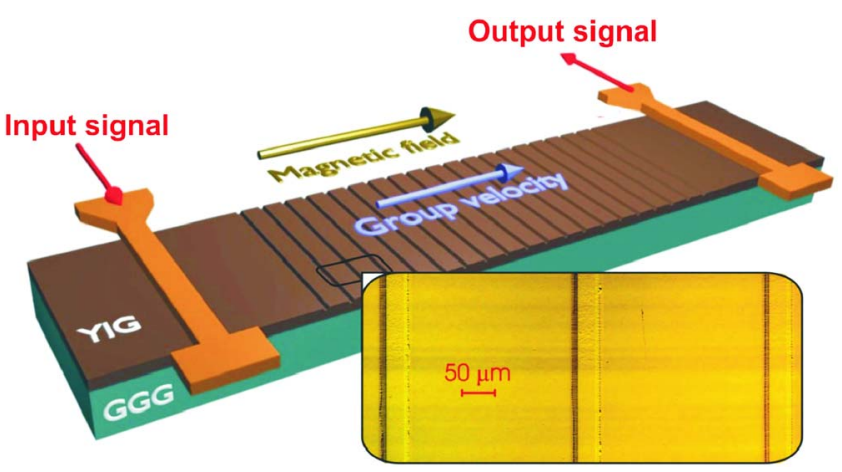

FIG. 1. (Color online) Sketch of the magnonic crystal structure used in the experiments. 

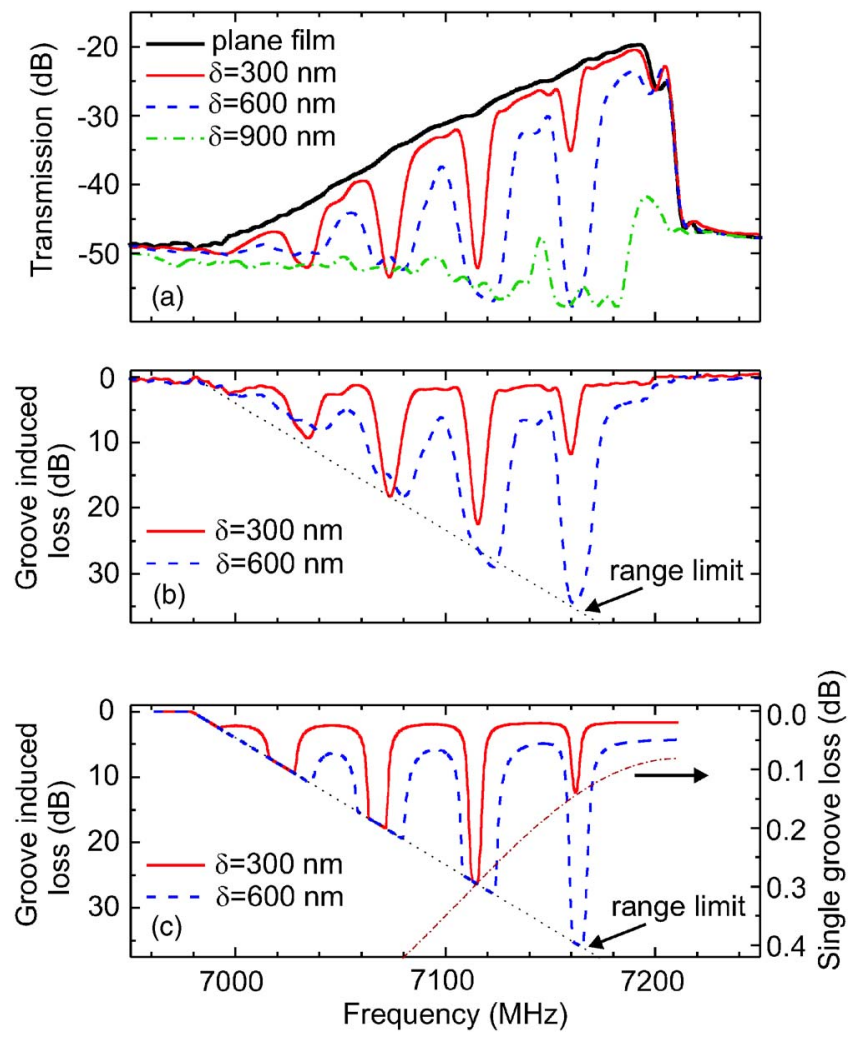

FIG. 2. (Color online) (a) BVMSW microwave transmission characteristics for an unstructured film (bold line) and for magnonic crystals for different groove depths $\delta$. (b) Experimentally measured loss induced by the grating. (c) Calculated loss. Parameters of calculation: groove number $N=20$, groove width at the bottom $w=30 \mu \mathrm{m}$, lattice constant $a=300 \mu \mathrm{m}$, film thickness $d_{0}=5.5 \mu \mathrm{m}$, saturation magnetization $4 \pi M_{0}=1750 \mathrm{G}$, bias magnetic field $H_{0}=1845 \mathrm{Oe}$, efficiency coefficient $\eta=6$, resonance linewidth $\Delta H=0.5$ Oe, and surface damage coefficient $\zeta=30$. The dash-dotted line shows the calculated loss induced by a single groove of $300 \mathrm{~nm}$ in depth.

In Fig. 2(b) one sees that the grooves as shallow as $300 \mathrm{~nm}$ result in the appearance of a set of rejection bands (or transmission gaps), where spin-wave transmission is highly reduced. According to the condition for Bragg reflection, higher-order rejection bands correspond to larger spinwave wave numbers. In the case of BVMSW the latter corresponds to lower frequencies. From the depths and the frequency widths $\Delta f$ of the gaps, one sees that the efficiency of the rejection increases with an increase in the order of Bragg reflection. This suggests that BVMSWs with smaller wavelengths are more sensitive to the introduced inhomogeneities.

Both Figs. 2(a) and 2(b) demonstrate that an increase in $\delta$ leads to an increase in the rejection efficiency and in $\Delta f$. A small frequency shift of the minima of transmission toward higher frequencies is observed, as well as an increase in insertion losses in the transmission (i.e., allowed) bands. For $\delta=900 \mathrm{~nm}$ the insertion loss in the whole spin-wave band is so important that almost no spin-wave propagation is observed [see Fig. 2(a)].

The points in Fig. 3 represent the insertion loss (upper panel) and the frequency width $\Delta f_{1}$ measured for the first transmission gap (the central frequency of the gap is $7160 \mathrm{MHz}$ ). This rejection band was chosen because of its largest experimental dynamic range. In Fig. 3(a) one sees that the difference in insertion loss for transmission and rejection bands can reach $30 \mathrm{~dB}$ for $\delta=0.5 \mu \mathrm{m}$. With an in-
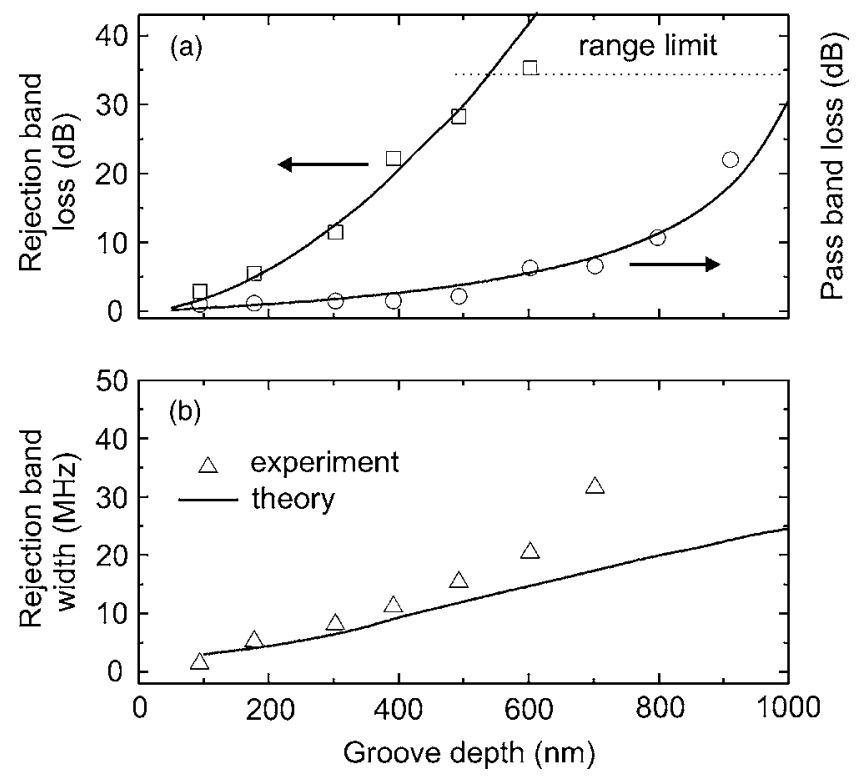

FIG. 3. (a) Insertion loss in rejection band (squares) and pass band (circles) as a function of groove depth; solid lines show corresponding calculated dependencies. (b) Experimental (triangles) and theoretical (line) first rejection bandwidths as a function of groove depth.

crease in the groove depth, the rejected power exceeds the dynamic range of the experimental setup, and the transmission loss cannot be measured. The observed frequency shift of the minimum of transmission with an increase in $\delta$ was 10-20 MHz. The maximum rejection bandwidth $\Delta f_{1}$ was almost $40 \mathrm{MHz}$.

A theoretical description of the scattering of dipole spin waves from inhomogeneities is given by a singular integral equation $\mathbf{m}(\mathbf{r})=4 \pi \hat{\kappa}\left[f, \mathbf{H}_{i}(\mathbf{r}), \mathbf{M}(\mathbf{r})\right] \cdot\left[\int_{V} \hat{G}\left(\mathbf{r}-\mathbf{r}^{\prime}\right) \mathbf{m}\left(\mathbf{r}^{\prime}\right) d \mathbf{r}^{\prime}\right.$ $+\mathbf{h}(\mathbf{r})$ ], where $\mathbf{m}$ is the dynamic magnetization, $\mathbf{h}$ is the microwave field of the input antenna, $\hat{G}\left(\mathbf{r}-\mathbf{r}^{\prime}\right)$ is the Green's function of the dipole magnetic field, and $\hat{\kappa}\left[f, \mathbf{H}_{i}(\mathbf{r}), \mathbf{M}(\mathbf{r})\right]$ is the tensor of microwave magnetic susceptibility, which depends on the spin-wave carrier frequency $f$ and on the magnetic parameters of the film. The latter are functions of the position $\mathbf{r}$ since the grooves induce inhomogeneity of the internal static field $\mathbf{H}_{i}$ and of the equilibrium magnetization $\mathbf{M}$. The integration is over the volume of the magnetic structure. Thus the inhomogeneity of film thickness is taken into account. The quasi-one-dimensional dipole field ${ }^{13}$ of the lowest BVMSW thickness mode decays at a distance of a few film thicknesses. Therefore, as the width of the grooves $w$ is much smaller than $a$, most of the distance between the consecutive grooves the spin wave travels is an eigenmode of a continuous film of thickness $d_{0}$. For these sections the integral equation reduces to a simple formula [see Eq. (50) in Ref. 14], which shows that between the grooves the transmitted and reflected waves only accumulate phase and decay (due to intrinsic magnetic damping). Thus, in order to describe the formation of stop bands, one has to consider scattering of a BVMSW from just one groove. The effect of a set of the grooves is obtained by cascading the structure periods using matrices of scattering $T$-parameters and taking interference effects into account.

Therefore in this work, instead of solving the singular integral equation, we considered the grating as a periodical sequence of sections of regular transmission lines with different propagation constants (different spin-wave wave num- 
bers) for the same carrier frequency. We neglect the fact that the groove edges are oblique and consider the groove cross section as a rectangle with the same depth and having the same area. The $T$-matrix $T^{(1)}$ for a section of an unstructured film of the length $a-w$ has diagonal components only: $T_{11}^{(1)}$ $=1 / T_{22}^{(1)}=e^{\left(-i k+k_{0}^{\prime \prime}\right)(a-w)}$, where $k$ is the spin-wave wave number in the unstructured film, $k_{0}^{\prime \prime}=\gamma \Delta H /\left(2 v_{\text {gr }}\right)$ is the rate of spin-wave spatial damping, $\gamma$ is the gyromagnetic ratio, $\Delta H$ is the ferromagnetic resonance linewidth, and $v_{\text {gr }}$ is the spinwave group velocity. Similarly, the $T$-matrix $T^{(3)}$ for a regular spin-wave film waveguide with the thickness $d=d_{0}-\delta$ is $T_{11}^{(3)}=1 / T_{22}^{(3)}=e^{\left(-i k+k_{\mathrm{g}}^{\prime \prime}\right) w d_{0} / d} ; T_{12}^{(3)}=T_{21}^{(3)}=0$, where $k_{g}^{\prime \prime}$ is the spinwave damping rate for the groove. Here we used the fact that the BVMSW dispersion law for small wave numbers $k d$ $\ll 1$ is practically linear; therefore the spin-wave wave number in the grooves is $k d_{0} / d$. To describe the increase in the loss in the pass bands with an increase in the groove depth, we introduce an empirical parameter $\zeta$ that accounts for a larger contribution of two-magnon scattering processes in the areas that underwent anisotropic etching. ${ }^{15}$ Then the damping rate in the grooves can be expressed as $k_{g}^{\prime \prime}=k_{0}^{\prime \prime}(1$ $\left.+\zeta \delta / d_{0}\right)$.

There are reflections from the junctions of the consecutive sections. The $T$-matrix for the front edge of the groove is $T^{(2)}$ and that for the rear edge is $T^{(4)}$. Therefore the $T$-matrix for one period of the structure is as follows: $T$ $=\left[T^{(1)} \cdot T^{(2)} \cdot T^{(3)} \cdot T^{(4)}\right]$. To obtain the $T$-matrix for the whole groove sequence, one has to raise $T$ to the $N$ th power. The reflection coefficient for a junction of two sections of regular waveguides is $\Gamma$. Following Ref. 16, the transmission coefficient through the junction is $1-\Gamma$. Then one obtains $T_{11}^{(2)}$ $=T_{22}^{(2)}=(1-\Gamma)^{-1}$ and $T_{12}^{(2)}=T_{21}^{(2)}=\Gamma(1-\Gamma)^{-1}$, and $T_{11}^{(4)}=T_{22}^{(4)}=(1$ $+\Gamma)^{-1}$ and $T_{12}^{(4)}=T_{21}^{(4)}=-\Gamma(1+\Gamma)^{-1}$.

Now one has to specify the form of the reflection coefficient $\Gamma$. Here we use the analogy of the change in the film waveguiding properties to a change in the characteristic impedance $Z$ of a microwave transmission line. ${ }^{17}$ We assume that the change in the characteristic impedance of a spinwave waveguide because of the change in YIG-film thickness is due to the change in the film effective inductance. Then the characteristic impedance is linearly proportional to the propagation constant (to the spin-wave wave number in our case), and from Eq. (3) in Ref. 16 we arrive at a formula for the reflection coefficient for the wave incident onto the edge of the groove from the unstructured section of the film: $\Gamma=\eta \delta /\left(2 d_{0}-\delta\right)$.

For the wave incident onto the same junction in the reverse direction, $\Gamma_{-}=-\Gamma$, which has been already taken into account in the expressions for the $T$-matrices above. The phenomenological parameter $\eta>1$ is introduced in this formula to account for eventual factors not taken into account in this simplistic model.

The results of our numerical computation of $T$ in the 20th power are shown in Figs. 2(c) and 3. One sees that this model is in qualitative agreement with all the tendencies we see in the experiment. In particular, it shows the observed increase in the rejection efficiency with an increase in $k$, and the correct behavior of all characteristics of the rejection bands as functions of groove depth $\delta$.

The formation of transmission gaps is due to multiple reflections from edges of all grooves which, form partial standing waves in the space between the grooves. The strength of these reflections is entirely determined by transmission and reflection of a single groove. The latter is shown (in a suitable scale) in Fig. 2(c) for $\delta=300 \mathrm{~nm}$ by a dashdotted continuous line. One sees that the peaks of rejection for the 20-groove structure follow this line. However, the quantitative agreement of this model is poor, unless one introduces a value of $\eta$ considerably larger than 1 (in our calculation $\eta=6$ ). In particular, for $\eta=1$, when the reflection from a groove edge is entirely due to the transformation of wave number, the model considerably underestimates the depth of transmission gaps. This suggests that other effects such as the transformation of the modal distribution of dynamic magnetization and the eventual generation of other thickness modes (and, possibly, back-transformation) through two-wave scattering processes give significant contributions to $\Gamma$.

In conclusion, in this work we experimentally demonstrated that in the BVMSW configuration a one-dimensional magnonic crystal showed excellent spin-wave signal rejection of more than $30 \mathrm{~dB}$. The width of the rejection bands exceeded the values for the other spin-wave configurations, ${ }^{3,6-8}$ and could be controlled by the groove depth. A simple model was proposed, which is in qualitative agreement with the experimental results.

Financial support by the DFG SE 1771/1-1, the Australian Research Council, and the University of Western Australia is acknowledged. Special acknowledgments are due to Dr. Sandra Wolff and the Nano+Bio Center, TU Kaiserslautern.

${ }^{1}$ Yu. V. Gulyayev and S. A. Nikitov, Dokl. Phys. 46, 687 (2001).

${ }^{2}$ P. A. Kolodin and B. Hillebrands, J. Magn. Magn. Mater. 161, 199 (1996).

${ }^{3}$ K. W. Reed, J. M. Owens, and R. L. Carter, Circuits Syst. Signal Process. 4, 157 (1985).

${ }^{4}$ R. W. Damon and J. R. Eshbach, Phys. Chem. Solids 19, 308 (1961).

${ }^{5}$ R. W. Damon and H. Van De Vaart, J. Appl. Phys. 36, 3453 (1965).

${ }^{6}$ C. G. Skyes, J. D. Adam, and J. H. Collins, Appl. Phys. Lett. 29, 388 (1976).

${ }^{7}$ J. P. Parekh and H. S. Tuan, Appl. Phys. Lett. 30, 667 (1977).

${ }^{8}$ J. P. Parekh and H. S. Tuan, IEEE Trans. Microwave Theory Tech. 26, 1039 (1978).

${ }^{9}$ A. A. Serga, B. Hillebrands, S. O. Demokritov, A. N. Slavin, P. Wierzbicki, V. Vasyuchka, O. Dzyapko, and A. Chumak, Phys. Rev. Lett. 94, 167202 (2005).

${ }^{10}$ S. O. Demokritov, A. A. Serga, V. E. Demidov, B. Hillebrands, M. Kostylev, and B. A. Kalinikos, Nature (London) 426, 159 (2003).

${ }^{11}$ Yu. V. Kobljanskyj, G. A. Melkov, A. A. Serga, V. S. Tiberkevich, and A. N. Slavin, Appl. Phys. Lett. 81, 1645 (2002).

${ }^{12}$ M. P. Kostylev, A. A. Serga, T. Schneider, B. Leven, and B. Hillebrands, Appl. Phys. Lett. 87, 153501 (2005).

${ }^{13}$ K. Yu. Guslienko, S. O. Demokritov, B. Hillebrands, and A. N. Slavin, Phys. Rev. B 66, 132402 (2002).

${ }^{14}$ M. P. Kostylev, A. A. Serga, T. Schneider, T. Neumann, B. Leven, B. Hillebrands, and R. L. Stamps, Phys. Rev. B 76, 184419 (2007).

${ }^{15}$ J. Basterfield, Br. J. Appl. Phys. 2, 1159 (1969).

${ }^{16}$ W. Berry, IEEE Trans. Microwave Theory Tech. 34, 80 (1986).

${ }^{17}$ B. A. Kalinikos, personal communication (1987). 
Applied Physics Letters is copyrighted by the American Institute of Physics (AIP). Redistribution of journal material is subject to the AIP online journal license and/or AIP copyright. For more information, see http://ojps.aip.org/aplo/aplcr.jsp 